\title{
Diferencias en el mecanismo y patrón lesional, gravedad y evolución de los pacientes politraumatizados en función del género
}

\author{
A. HERNÁNDEZ-TEJEDOR, C. GARCÍA-FUENTES, D. TORAL-VÁZQUEZ, \\ M. CHICO-FERNÁNDEZ Y E. ALTED-LÓPEZ \\ Servicio de Medicina Intensiva. Sección de Politrauma y Emergencias. \\ Hospital Universitario 12 de Octubre. Madrid. España.
}

Objetivo. Estudiar el valor pronóstico y la diferencia entre géneros de los mecanismos y patrones lesionales de los pacientes politraumatizados.

Diseño. Estudio observacional descriptivo prospectivo.

Ámbito. Unidad de Cuidados Intensivos (UCI) de Politrauma de un hospital terciario con helisuperficie.

Pacientes. Novecientos cincuenta y tres pacientes ingresados consecutivamente en la $\mathrm{UCI}$ de Politrauma entre 2003 y 2005.

Variables de interés principales. Describimos el patrón lesional según el MAIS de cada región anatómica y empleamos el Injury Severity Score (ISS) para estimar la gravedad y la metodología Trauma Injury Severity Score para calcular la probabilidad de supervivencia al ingreso.

Resultados. La relación hombres:mujeres fue de $4: 1$ con una edad media de $38 \pm 16$ años. Más del $50 \%$ de los ingresos fueron por accidente de tráfico. Las regiones anatómicas de la Abbreviated Injury Scale en las que hubo diferencias estadísticamente significativas entre géneros fueron la 1, la 6 y la 8 . El valor MAIS1 fue mayor de 4 en el $21,3 \%$ de los hombres y en el $28,3 \%$ de las mujeres ( $p<0,05)$; el MAIS6 fue mayor de 2 en el $9,9 \%$ de los hombres y en el $16,7 \%$ de las mujeres ( $p<0,01$ ); el MAIS 8 fue mayor de 2 en el $23,8 \%$ de los hombres y en el $31,8 \%$ de las mujeres $(p<$

Correspondencia: Dr. A. Hernández-Tejedor.

UCI de Politrauma y Emergencias.

Hospital Universitario 12 de Octubre.

Avda. Córdoba, s/n.

28041 Madrid. España.

Correo electrónico: albertohmed@ hotmail.com

Manuscrito aceptado el 18-I-2008.
$0,05)$. La supervivencia al alta de la UCI fue del $85,5 \%$ para los hombres y del $76,8 \%$ para las mujeres $(p<0,01)$. EI ISS medio fue de $23,9(23,2$ para los hombres y 26,5 para las mujeres [p $<0,01]$ ).

Conclusión. La mortalidad ajustada a gravedad al ingreso no está influida por el género. Las mujeres suponen sólo el $20 \%$ de los ingresos pero presentan mayor gravedad y más lesiones craneoencefálicas, y éstas son más graves. Las diferencias en el mecanismo y el patrón lesional entre hombres y mujeres son escasas.

PALABRAS CLAVE: diferencias por género, índices de gravedad en trauma, pronóstico, trauma, mecanismo lesional, patrón le sional.

DIFFERENCES IN INJURY MECHANISM AND PATTERN, SEVERITY AND OUTCOME OF MULTIPLE TRAUMA PATIENTS DEPENDING ON GENDER

Objective. To study de prognostic value and the difference between genders in the injury mechanism and pattern of multiple trauma patients.

Design. Prospective descriptive observational study.

Setting. Trauma Intensive Care Unit (ICU) of a tertiary hospital equipped with an heliport.

Patients. 953 patients consecutively admitted to ICU between 2003 and 2005.

Main variables of interest. We describe the injury pattern with MAIS of each anatomical region and use Injury Severity Score (ISS) to estimate severity and Trauma Injury Severity Score (TRISS) methodology to calculate survival probability at admission.

Results. Ratio male:female was $4: 1$, with a mean age of $38 \pm 16$ years. More than $50 \%$ of patients were injured in a traffic accident. MAIS1 had a va- 
lue of more than 4 in $21.3 \%$ of males and in $28.3 \%$ of females ( $p<0.05)$; MAIS6 was more than 2 in $9.9 \%$ of males and in $16.7 \%$ of females $(p<0.01)$; MAIS8 was greater than 2 in $23,8 \%$ of males and in $31.8 \%$ of females $(p<0.05)$. Survival at discharge from ICU was $85.5 \%$ for males and $76.8 \%$ for females ( $p$ < 0.01). Mean ISS was 23.9 (23.2 for males and 26.5 for females, $p<0.01$ ).

Conclussion. Gender does not play a role in mortality adjusted to severity at admission. Women are one fifth of all admissions but are more severe and suffer more craniocerebral injuries and these are more severe. There are few differences in injury mechanism and pattern between men and women.

KEY WORDS: gender differences, trauma severity indices, outcome, trauma, injury mechanism, injury pattern.

\section{INTRODUCCIÓN}

La influencia del género en el patrón lesional y en la evolución de los pacientes politraumatizados no es bien conocida. Tampoco se conoce la influencia del patrón lesional sobre la supervivencia.

Dado que gran parte de los traumatismos se producen en ambientes y circunstancias en las que se ponen de manifiesto diferencias en el comportamiento entre hombres y mujeres, como pueden ser el ocio, el medio laboral o el empleo de vehículos a motor, es posible también que el género se asocie con determinados patrones lesionales, gravedad o necesidades asistenciales.

En los últimos años se han publicado diversos estudios que comparan la supervivencia de los pacientes que ingresan en los hospitales en general y en los Servicios de Medicina Interna (SMI) en particular, en función del género y la mayoría de ellos apunta que la supervivencia es mayor en mujeres ${ }^{1,2}$. Se han elaborado numerosas teorías, generalmente en torno a diferencias hormonales o inmunitarias ${ }^{3,4}$. También se han publicado estudios similares en poblaciones de pacientes politraumatizados, con resultados dispares, si bien la mayoría concluye que la mortalidad global no está influida por el género ${ }^{5,6}$. Sin embargo, se han realizado pocos estudios en los que se compare el mecanismo lesional de hombres y mujeres.

La hipótesis de trabajo es que el género influye en el mecanismo lesional, y éste en el patrón lesional, en la gravedad y en la evolución clínica. El objetivo de este estudio es conocer los mecanismos lesionales de los pacientes politraumatizados y sus patrones, tratando de demostrar sus diferencias entre hombres y mujeres, así como su relación con la gravedad y la evolución de los pacientes.

\section{PACIENTES Y MÉTODO}

Realizamos un estudio observacional descriptivo en el que incluimos a todos los pacientes traumáticos in-
TABLA 1. Regiones anatómicas de la Abbreviated Injury Scale

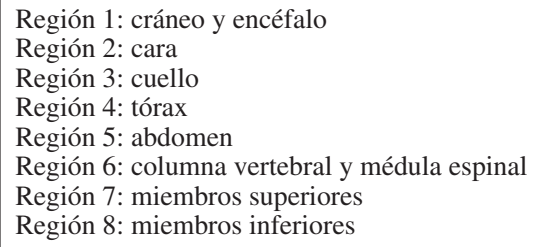

gresados en la Unidad de Cuidados Intensivos (UCI) de Politrauma y Emergencias de nuestro hospital, un hospital terciario con helisuperficie, durante el periodo comprendido entre los años 2003 y 2005. Revisamos los datos recogidos prospectivamente en nuestra base de datos. Incluimos un total de 953 pacientes.

Los datos sobre el mecanismo lesional se han obtenido de los informes de asistencia de los Servicios de Urgencias prehospitalarios. Para describir el patrón lesional empleamos el código de gravedad de la lesión más grave de cada región anatómica de la Abbreviated Injury Scale (AIS) del año 19907, al que denominamos MAISn, siendo $\mathrm{n}$ la región anatómica. La lista de regiones anatómicas de la AIS aparece en la tabla 1. Para cada región anatómica establecemos un punto de corte entre lesiones consideradas menores (puntuación de gravedad inferior o igual a 2) y mayores (puntuación superior o igual a 3); en la región 1 (cráneo y encéfalo) establecemos una clasificación adicional de lesiones muy graves (las que tienen una puntuación superior o igual a 5). Las lesiones no diagnosticadas anatómicamente, generalmente por fallecimiento del paciente antes de poder realizar pruebas complementarias de imagen que permitan su diagnóstico, no han sido consideradas.

Valoramos la gravedad del paciente al ingreso mediante el empleo del Injury Severity Score (ISS) ${ }^{8}$ y el Revised Trauma Score (RTS).

También se estudiaron las siguientes actitudes terapéuticas: intubación orotraqueal, transfusión de concentrados de hematíes, necesidad de cirugía urgente y días de ventilación mecánica.

Calculamos al ingreso la probabilidad de supervivencia por el método Trauma Injury Severity Score $(\text { TRISS })^{10}$. Finalmente se estudiaron la estancia y la evolución de los pacientes, y se calculó la probabilidad de supervivencia de acuerdo a la gravedad al ingreso.

\section{Análisis estadístico}

Para la comparación de variables cuantitativas se utilizó la t de Student y para las variables cualitativas se empleó la Chi cuadrado. Las variables cuantitativas continuas se expresan como media \pm desviación estándar y las cualitativas como porcentaje.

\section{RESULTADOS}

De los 953 pacientes ingresados, $758(79,5 \%)$ fueron hombres. La edad fue de $38 \pm 16$ años $(37,2$ 
TABLA 2. Distribución en función de la situación en que se produjo el traumatismo

\begin{tabular}{|l|c|c|c|c|}
\hline & $\begin{array}{c}\text { Total } \\
(\mathrm{n}=942)^{\mathrm{a}}\end{array}$ & $\begin{array}{c}\text { Hombres } \\
(\mathrm{n}=747)\end{array}$ & $\begin{array}{c}\text { Mujeres } \\
(\mathrm{n}=195)\end{array}$ & $\mathrm{p}$ \\
\hline Accidente de tráfico & $535(56,8 \%)$ & $417(55,8 \%)$ & $118(60,5 \%)$ & $\mathrm{p}=0,24$ \\
Accidente laboral & $120(12,7 \%)$ & $119(15,9 \%)$ & $1(0,5 \%)$ & $\mathrm{p}<0,0001$ \\
Agresión & $107(11,4 \%)$ & $87(11,6 \%)$ & $20(10,3 \%)$ & $\mathrm{p}=0,59$ \\
Lesión accidental & $75(8 \%)$ & $52(7 \%)$ & $23(11,8 \%)$ & $\mathrm{p}<0,05$ \\
Lesiones autoinfligidas & $49(5,2 \%)$ & $32(4,3 \%)$ & $17(8,7 \%)$ & $\mathrm{p}<0,05$ \\
Accidente deportivo & $35(3,7 \%)$ & $28(3,7 \%)$ & $7(3,6 \%)$ & $\mathrm{p}=0,92$ \\
Atentado & $9(1 \%)$ & $4(0,5 \%)$ & $5(2,6 \%)$ & $\mathrm{p}<0,01$ \\
Otros & $12(1,3 \%)$ & $8(1,1 \%)$ & $4(2,1 \%)$ & $\mathrm{p}=0,28$ \\
\hline
\end{tabular}

aFalta información en 11 de los 953 incluidos en el estudio.

\pm 15,6 para los hombres y 40,9 $\pm 19,2$ para las mujeres).

En cuanto a la situación en que se produjo el traumatismo, más de la mitad fueron consecuencia de un accidente de tráfico, como se muestra en la tabla 2. Únicamente 1 de los 120 pacientes ingresados por accidente laboral fue mujer. El mecanismo lesional en la población global del estudio y su distribución de acuerdo al género se muestra en la tabla 3. El más frecuente, tanto en hombres como en mujeres, fue el accidente de coche.

La gravedad de las lesiones que presentaron los pacientes en las diferentes regiones de la escala AIS, valoradas a través del valor MAISn, se muestra en la tabla 4. En las regiones en que se hallaron diferencias significativas entre hombres y mujeres (cráneo y encéfalo, raquimedular y miembros inferiores) la distribución de los valores MAISn fue la que aparece en la figura 1. Las lesiones en las regiones anteriormente mencionadas tuvieron una mayor frecuencia relativa y mayor gravedad en mujeres.

En la tabla 5 podemos ver que no se encontraron diferencias estadísticamente significativas en las actitudes terapéuticas empleadas en hombres y en mujeres. Únicamente hay una tendencia no significativa en el volumen de concentrados de hematíes infundidos $(947 \pm 122 \mathrm{ml}$ en mujeres frente a $706 \pm 58 \mathrm{ml}$ en varones $(\mathrm{p}=0,06)$.

Al alta de la UCI, la supervivencia fue del 85,5\% para los hombres y del $76,8 \%$ para las mujeres ( $\mathrm{p}<$ $0,01)$.

Según había sido diseñado el estudio, calculamos la probabilidad de supervivencia de los pacientes en el momento del ingreso según la metodología TRISS. El ISS medio de los pacientes incluidos fue de 23,9 (23,2 para los hombres y 26,5 para las mujeres; $\mathrm{p}<$ 0,01). El RTS medio de los pacientes fue de 10,1 (10,2 para los hombres y 9,8 para las mujeres; $\mathrm{p}=$ 0,13). Los cálculos TRISS ofrecen los siguientes valores: la probabilidad de supervivencia al ingreso para los hombres fue del $83,1 \%$ y para las mujeres del $74,5 \%(\mathrm{p}<0,001)$. La supervivencia real fue un $2,9 \%$ superior a la esperada en el caso de los hombres y un $3,1 \%$ superior en el caso de las mujeres y no se encuentran diferencias estadísticamente significativas en este campo.

En cuanto a la estancia, esta fue de 5,98 \pm 0,33 días para los hombres y de $6,53 \pm 0,73$ días para las mujeres $(p=0,47)$.

\section{DISCUSIÓN}

En nuestro estudio, la mortalidad ajustada a gravedad al ingreso no presentó diferencias en función del género. Este resultado es congruente con otros estudios recientes que concluyen que la mortalidad global en pacientes politraumatizados no está influida por el género $^{5,6,11-18}$.

En consonancia con los resultados de estudios publicados en los últimos años que muestran que el género femenino podría ser un factor de protección en casos de enfermedad grave ${ }^{3,4,19,20}$, quizá por las diferencias hormonales y/o inmunológicas entre hombres y mujeres, en los SMI polivalentes las mujeres presentan menor mortalidad ajustada por gravedad al ingreso $^{1,2,21-23}$. Sin embargo, estos datos no se extienden

TABLA 3. Distribución en función del mecanismo lesional

\begin{tabular}{|l|c|c|c|}
\hline & $\begin{array}{c}\text { Total } \\
(\mathrm{n}=943)^{\mathrm{a}}\end{array}$ & $\begin{array}{c}\text { Hombres } \\
(\mathrm{n}=748)\end{array}$ & $\begin{array}{c}\text { Mujeres } \\
(\mathrm{n}=195)\end{array}$ \\
\hline Accidente de coche & $303(32,1 \%)$ & $222(29,7 \%)$ & $81(41,6 \%)$ \\
Accidente de moto & $120(12,7 \%)$ & $110(14,7 \%)$ & $10(5,1 \%)$ \\
Atropello & $82(8,7 \%)$ & $58(7,8 \%)$ & $24(12,3 \%)$ \\
Precipitación & $134(14,2 \%)$ & $111(14,8 \%)$ & $23(11,8 \%)$ \\
Caída accidental & $45(4,8 \%)$ & $31(4,1 \%)$ & $14(7,2 \%)$ \\
Arma blanca & $73(7,8 \%)$ & $65(8,7 \%)$ & $8(4,1 \%)$ \\
Arma de fuego & $25(2,7 \%)$ & $21(2,8 \%)$ & $4(2,1 \%)$ \\
Otros & $161(17,1 \%)$ & $130(17,4 \%)$ & $31(15,9 \%)$ \\
$\mathrm{p}=0,005$ & $\mathrm{p}<0,05$ \\
$\mathrm{p}=0,56$ & $\mathrm{p}=0,62$ \\
\hline
\end{tabular}

aFalta información en 10 de los 953 pacientes incluidos en el estudio. 
HERNÁNDEZ-TEJEDOR A ET AL. DIFERENCIAS EN EL MECANISMO Y PATRÓN LESIONAL, GRAVEDAD Y EVOLUCIÓN DE LOS PACIENTES POLITRAUMATIZADOS EN FUNCIÓN DEL GÉNERO

TABLA 4. Distribución en función del mecanismo lesional expresado a través del valor MAISn

\begin{tabular}{|c|c|c|c|c|}
\hline & $\begin{array}{c}\text { Total } \\
(\mathrm{n}=922)^{\mathrm{a}}\end{array}$ & $\begin{array}{c}\text { Varones } \\
(\mathrm{n}=731)\end{array}$ & $\begin{array}{c}\text { Mujeres } \\
(\mathrm{n}=191)\end{array}$ & $\mathrm{p}$ \\
\hline \multicolumn{5}{|l|}{ MAIS1 } \\
\hline $0-2$ & $526(57 \%)$ & $426(58,3 \%)$ & $100(52,3 \%)$ & \multirow[t]{3}{*}{$\mathrm{p}<0,05$} \\
\hline $3-4$ & $186(20,2 \%)$ & $149(20,4 \%)$ & $37(19,4 \%)$ & \\
\hline $5-6$ & $210(22,8 \%)$ & $156(21,3 \%)$ & $54(28,3 \%)$ & \\
\hline \multicolumn{5}{|l|}{ MAIS2 } \\
\hline $0-2$ & $847(91,3 \%)$ & $669(90,7 \%)$ & $178(93,2 \%)$ & \multirow[t]{2}{*}{$\mathrm{p}=0,29$} \\
\hline$>2$ & $81(8,7 \%)$ & $68(9,3 \%)$ & $13(6,8 \%)$ & \\
\hline \multicolumn{5}{|l|}{ MAIS3 } \\
\hline $0-2$ & $920(98,8 \%)$ & $729(98,6 \%)$ & $191(99,5 \%)$ & \multirow[t]{2}{*}{$\mathrm{p}=0,34$} \\
\hline$>2$ & $11(1,2 \%)$ & $10(1,4 \%)$ & $1(0,5 \%)$ & \\
\hline \multicolumn{5}{|l|}{ MAIS4 } \\
\hline $0-2$ & $574(61,7 \%)$ & $460(62,1 \%)$ & $114(60 \%)$ & \multirow[t]{2}{*}{$\mathrm{p}=0,60$} \\
\hline$>2$ & $357(38,3 \%)$ & $281(37,9 \%)$ & $76(40 \%)$ & \\
\hline \multicolumn{5}{|l|}{ MAIS5 } \\
\hline $0-2$ & $797(86,2 \%)$ & $635(86,2 \%)$ & $162(86,2 \%)$ & \multirow[t]{2}{*}{$\mathrm{p}=1$} \\
\hline$>2$ & $128(13,8 \%)$ & $102(13,8 \%)$ & $26(13,8 \%)$ & \\
\hline \multicolumn{5}{|l|}{ MAIS6 } \\
\hline $0-2$ & $825(88,7 \%)$ & $665(90,1 \%)$ & $160(83,3 \%)$ & \multirow[t]{2}{*}{$\mathrm{p}<0,01$} \\
\hline$>2$ & $105(11,3 \%)$ & $73(9,9 \%)$ & $32(16,7 \%)$ & \\
\hline \multicolumn{5}{|l|}{ MAIS7 } \\
\hline $0-2$ & $812(87,3 \%)$ & $645(87,4 \%)$ & $167(87 \%)$ & \multirow[t]{2}{*}{$\mathrm{p}=0,88$} \\
\hline$>2$ & $118(12,7 \%)$ & $93(12,6 \%)$ & $25(13 \%)$ & \\
\hline \multicolumn{5}{|l|}{ MAIS8 } \\
\hline $0-2$ & $694(74,5 \%)$ & $563(76,2 \%)$ & $131(68,2 \%)$ & \multirow[t]{2}{*}{$\mathrm{p}<0,05$} \\
\hline$>2$ & $237(25,5 \%)$ & $176(23,8 \%)$ & $61(31,8 \%)$ & \\
\hline \multicolumn{5}{|l|}{ MAIS9 } \\
\hline $0-2$ & $924(99,4 \%)$ & $735(99,6 \%)$ & $189(98,4 \%)$ & \multirow[t]{2}{*}{$\mathrm{p}=0,07$} \\
\hline$>2$ & $6(0,6 \%)$ & $3(0,4 \%)$ & $3(1,6 \%)$ & \\
\hline
\end{tabular}

${ }^{\mathrm{a} F a l t a ~ i n f o r m a c i o ́ n ~ e n ~} 31$ de los 953 pacientes incluidos en el estudio.

a pacientes politraumatizados, y tampoco en nuestra serie se aprecia esta tendencia.

Sin embargo, hallamos diferencias en la gravedad de los pacientes. Según nuestra hipótesis de trabajo, las diferencias en la conducta de hombres y mujeres podrían dar lugar a una gravedad diferente. A modo de ejemplo, conocemos por datos publicados por la Dirección General de Tráfico ${ }^{24}$ que los conductores varones se ven involucrados en accidentes de tráfico más graves, lo que podría dar lugar a diferencias en el patrón lesional en los accidentes de tráfico entre hombres y mujeres, así como a una mayor necesidad de intervenciones quirúrgicas y una evolución diferente.

El dato más significativo es que la población femenina que ingresa en nuestra Unidad presenta mayor gravedad que la masculina. La principal diferencia, clínicamente relevante, es la presencia de lesio- nes más graves en el cráneo y el encéfalo en mujeres.

Uno de los factores que podrían influir en la gravedad de los pacientes es su diferente patrón, hecho que hasta ahora no ha sido estudiado en profundidad.

Son escasas las diferencias en el mecanismo lesional entre hombres y mujeres, y probablemente esto se relacione con las mínimas diferencias en el patrón de las lesiones, a diferencia de lo que intentábamos demostrar.

En nuestra serie, el hecho de que de los 120 accidentes laborales sólo uno fuera sufrido por una mujer es un hecho destacado; la justificación probablemente la hallemos en las características poblacionales de esta zona del sur de Madrid; esto podría haber dado lugar a diferencias en el patrón lesional o incluso en la mortalidad; no obstante, la mortalidad de este subgrupo fue del $11,67 \%$, sólo ligeramente superior a la media de nuestra población.

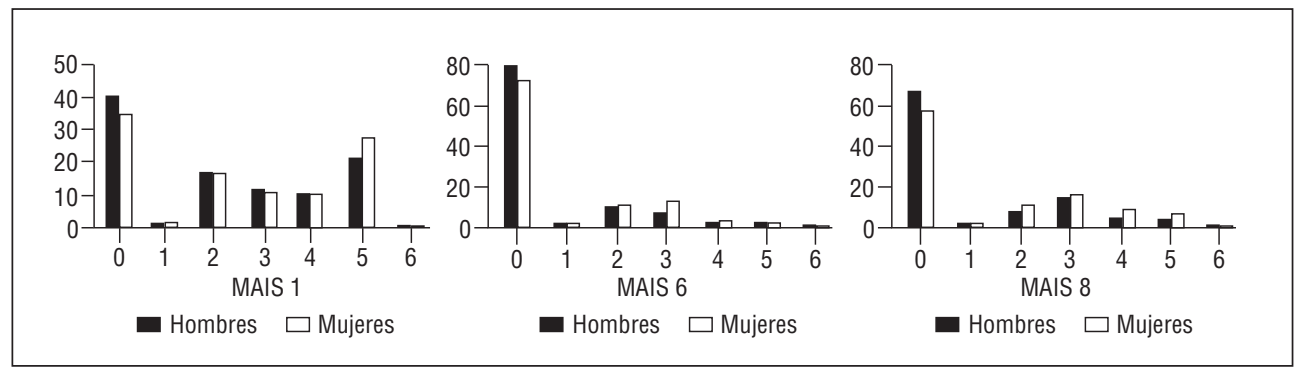

Figura 1. Distribución del valor MAIS de las regiones anatómicas 1, 6 y 8 en función del género. 
TABLA 5. Actitudes terapéuticas empleadas ${ }^{a}$

\begin{tabular}{|c|c|c|c|}
\hline & $\begin{array}{c}\text { Varones } \\
(\mathrm{n}=689)\end{array}$ & $\begin{array}{c}\text { Mujeres } \\
(\mathrm{n}=186)\end{array}$ & $\mathrm{p}$ \\
\hline Volumen de concentrados de hematíes transfundidos (ml) & $706 \pm 58$ & $947 \pm 122$ & $\mathrm{p}=0,06$ \\
\hline Tiempo de ventilación mecánica (días) & $4,05 \pm 0,27$ & $4,45 \pm 0,60$ & $\mathrm{p}=0,51$ \\
\hline Intubación orotraqueal & $271(38,4 \%)$ & $80(42,6 \%)$ & $\mathrm{p}=0,30$ \\
\hline Cirugía urgente & $253(35,8 \%)$ & $68(36 \%)$ & $\mathrm{p}=0,96$ \\
\hline
\end{tabular}

aFalta información en 78 de los 953 pacientes incluidos en el estudio.

Las únicas lesiones que presentan una mayor frecuencia en mujeres con significación estadística son las lesiones accidentales (caídas y otras lesiones casuales) y las autoinfligidas.

Uno de los posibles sesgos que haya podido influir en estos datos es la falta de datos sobre las lesiones de los pacientes que fallecieron en los primeros minutos $\mathrm{u}$ horas de ingreso.

Se necesita realizar nuevos estudios en otras poblaciones para estudiar la influencia del género en el patrón lesional de los politraumatizados graves.

\section{Declaración de conflicto de intereses}

Los autores han declarado no tener ningún conflicto de intereses.

\section{BIBLIOGRAFÍA}

1. Gordon HS, Rosenthal GE. The relationship of gender and inhospital death: increased risk of death in men. Med Care. 1999;37: 318-24.

2. Kollef MH, O'Brien JD, Silver P. The impact of gender on outcome from mechanical ventilation. Chest. 1997;111:434-41.

3. Angele MK, Ayala A, Monfils BA, Cioffi WG, Bland KI, Chaudry IH. Testosterone and/or low estradiol: normally required but harmful immunologically for males after trauma-hemorrhage. J Trauma. 1998;44:78-85.

4. Roof RL, Hall ED. Gender differences in acute CNS trauma and stroke: neuroprotective effects of estrogen and progesterone. $\mathrm{J}$ Neurotrauma. 2000;17:367-88.

5. Rappold JF, Coimbra R, Hoyt DB, Potenza BM, Fortlage D, Holbrook T. Female gender does not protect blunt trauma patients from complications and mortality. J Trauma. 2002;53:436-41.

6. Mostafa G, Huynh T, Sing RF, Miles WS, Norton HJ, Thomason MH. Gender-related outcomes in trauma. J Trauma. 2002; 53:430-5.

7. Association for the Advancement of Automotive Medicine. The Abbreviated Injury Scale, 1990 Revision. Des Plaines, Illinois: Association for the Advancement of Automotive Medicine; 1990.

8. Baker SP, O’Neill B, Haddon W Jr, Long WB. The injury severity score: a method for describing patients with multiple injuries and evaluating emergency care. J Trauma. 1974;14:187-96.
9. Champion HR, Sacco WJ, Copes WS, Gann DS, Gennarelli TA, Flanagan ME. A revision of the trauma score. J Trauma. 1989; 29:623-9.

10. Boyd CR, Tolson MA, Copes WS. Evaluating trauma care: the TRISS method. J Trauma. 1987;27:370-8.

11. Gannon CJ, Napolitano LM, Pasquale M, Tracy JK, McCarter RJ. A statewide population-based study of gender differences in trauma: validation of a prior single-institution study. J Am Coll Surg. 2002;195:11-8.

12. Holbrook TL, Hoyt DB, Anderson JP. The importance of gender on outcome after major trauma: functional and psychologic outcomes in women versus men. J Trauma. 2001:50:270-3.

13. Davis DP, Douglas DJ, Smith W, Sise MJ, Vilke GM, Holbrook TL, et al. Traumatic brain injury outcomes in pre- and post-menopausal females versus age-matched males. J Neurotrauma. 2006;23:140-8

14. George RL, McGwin G, Metzger J, Chaudry IH, Rue LW. The association between gender and mortality among trauma patients as modified by age. J Trauma. 2003;54:464-71.

15. Napolitano LM, Greco ME, Rodríguez A, Kufera JA, West RS, Scalea TM. Gender differences in adverse outcomes after blunt trauma. J Trauma. 2001;50:274-80.

16. Evans L. Female compared with male fatality risk from similar physical impacts. J Trauma. 2001;50:281-8.

17. Coimbra R, Hoyt DB, Potenza BM, Fortlage D, Hollingsworth-Fridlund P. Does sexual dimorphism influence outcome of traumatic brain injury patients? The answer is no! J Trauma 2003;54:689-700

18. George RL, McGwin G, Windham ST, Melton SM, Metzger J, Chaudry IH, et al. Age-related gender differential in outcome following blunt trauma or penetrating trauma. Shock. 2002;18:311-5.

19. Wichmann MW, Zellweger R, DeMaso CM, Ayala A, Chaudry IH. Mechanism of immunosuppression in males following trauma-hemorrhage. Critical role of testosterone. Arch Surg. 1996;131:1186-91.

20. Roof RL, Hall ED. Estrogen-related gender differences in survival rate, and cortical blood flow after impact-acceleration head injury in rats. J Neurotrauma. 2000;17:1155-69.

21. Ziser A, Plevak DJ, Wiesner RH, Rakela J, Offord KP, Brown DL. Morbidity and mortality in cirrhotic patients undergoing anesthesia and surgery. Anesthesiology. 1999;90:42-53.

22. Weaver WD, White HD, Wilcox RG, Ayward PE, Morris D, Guerci A, et al. Comparisons of characteristics and outcomes among women and men with acute myocardial infarction treated with thrombolytic therapy. JAMA. 1996;275:777-82.

23. Wohltmann CD, Spain DA, Carrillo EH, Boaz PW, Aluchette F, Kearney PA. Does gender affect outcome in trauma patients? Crit Care Med. 1999;27 Suppl:A176.

24. Anuario estadístico de accidentes 2004. Madrid: DGT; 2005. 